\title{
PerformanceAnalysisof a StandbySystemusing Exponential-Rayleigh-Weibull Distributions
}

\section{R.K. Bhardwaj ${ }^{1}$, Komaldeep Kaur ${ }^{2}$}

\author{
${ }^{1}$ Department of Statistics, Punjabi University Patiala-147002, India \\ ${ }^{2}$ Department of Statistics, M. S. G.C., University of Delhi- , India \\ rkb_mstates@rediffmail.com
}

Article History: Received: 11 January 2021; Accepted: 27 February 2021; Published online: 5 April 2021

\begin{abstract}
All through the life-cycle of a standby system, it is verychallenging to keep a standby unit workable.It may be fatalif the standby found non-workablewhen needed.This paper evaluates the performance of a standby systemworking under two primary constraintsby underlining the condition of the spare unit in standby mode. The first constraint is the maximum redundancy time for the standby and the second is maximum operation time for the operating unit.The standby unit failson exceeding the maximum time threshold andthereafterthe decision about its repair/replacement is subjected tothe inspection. While after surpassing the maximum operating time limit preventive maintenance is carried outfor the operating unit.To study the long-run performance or life-cycle of the system various performance indices have been analyzed usingthe theory of discrete-state continuous-time semi-Markov regenerative processes.Exponential, Rayleigh and Weibull probability distributions are used to study the system performance numerically.
\end{abstract}

Keyword:Semi-Markov, Cold-Standby System, Probabilistic Model, Steady State.

\section{Introduction}

In everyday life, we come across many systems equipped with redundant units to facilitate the smooth functioning andensuringhigheravailability and system reliability. For instance, the spare engine in a jet fighter,alternate power supply in an Intensive Care Units, redundant safety installation in an atomic power plant, parallel lines in a communication network, parallel service counter in a bankand many more.Though the provision of a spare componentguarantees reliability, availability and even safety in some cases but side by side put challenges to the budgetary resources. Despite all financial obligations, however, there are many safety installations where risk cannot be taken with reliability, in any case.Therefore, the provision of standby remains popular among safety and reliability professionals [ see [1], [2], [3], [4], [5]]. An essential thing about a standby system is that it can be restored if and only if the spare unit found perfectly operable. In all the above studies, it is commonly assumed that the standby unit always found operable when needed.Is it practically correct? There is no doubt that the active operating load on standby unit in a cold-standby system is not equal to that of an operating unit, but this factor alone cannot be assumed responsible for the current state of standby. Indeed, the state of standby depends firstly onthe local environment andsecondly on the ways of its handling[[6], [7]].So depending upon such factors the standby unit may or may not found operable. If not thenthere would be adverse consequences. Therefore, the study of standby systems with the possibility of standby failure becomesvery muchsignificant.Earlier, this issuehasbeen discussedhardly [[8], [9], [10] ].Furthermore,no preventive measures are takenbeforethe system failure. It is either repaired or replaced at itsfailure. In particular, the preventive maintenance plan can better improve the system performance [[11], [12], [13]]. Keeping this fact in view this paper analyzes a stochastic model of a cold standby system incorporating the idea of pre-failure preventive maintenance of the operative unit after crossing a pre-specified time limit, termed as maximum operation time.This paper presents the analysis of a two-unit cold standby system using the theory of discrete-state continuous-time Markov regenerative processes[[14], [15], [16]]. The system works under two constraints namely maximum redundancy time for the standby unit and maximum operation time for the operative unit. Upon crossing a pre-defined threshold time limit the standby fails and passes through inspection for deciding about its repair or replacement whereas the operative unit be given preventive maintenance aiming at enhancing system performance. The practical importance of theoretical results is shown in a particular case using Weibull distribution [[17],[18]].

\section{Acronyms and Notations}
$E / \bar{E}$
$U / \bar{U}$
: The set of regenerative/ Non-regenerative states
: The set of up-states/ down states 


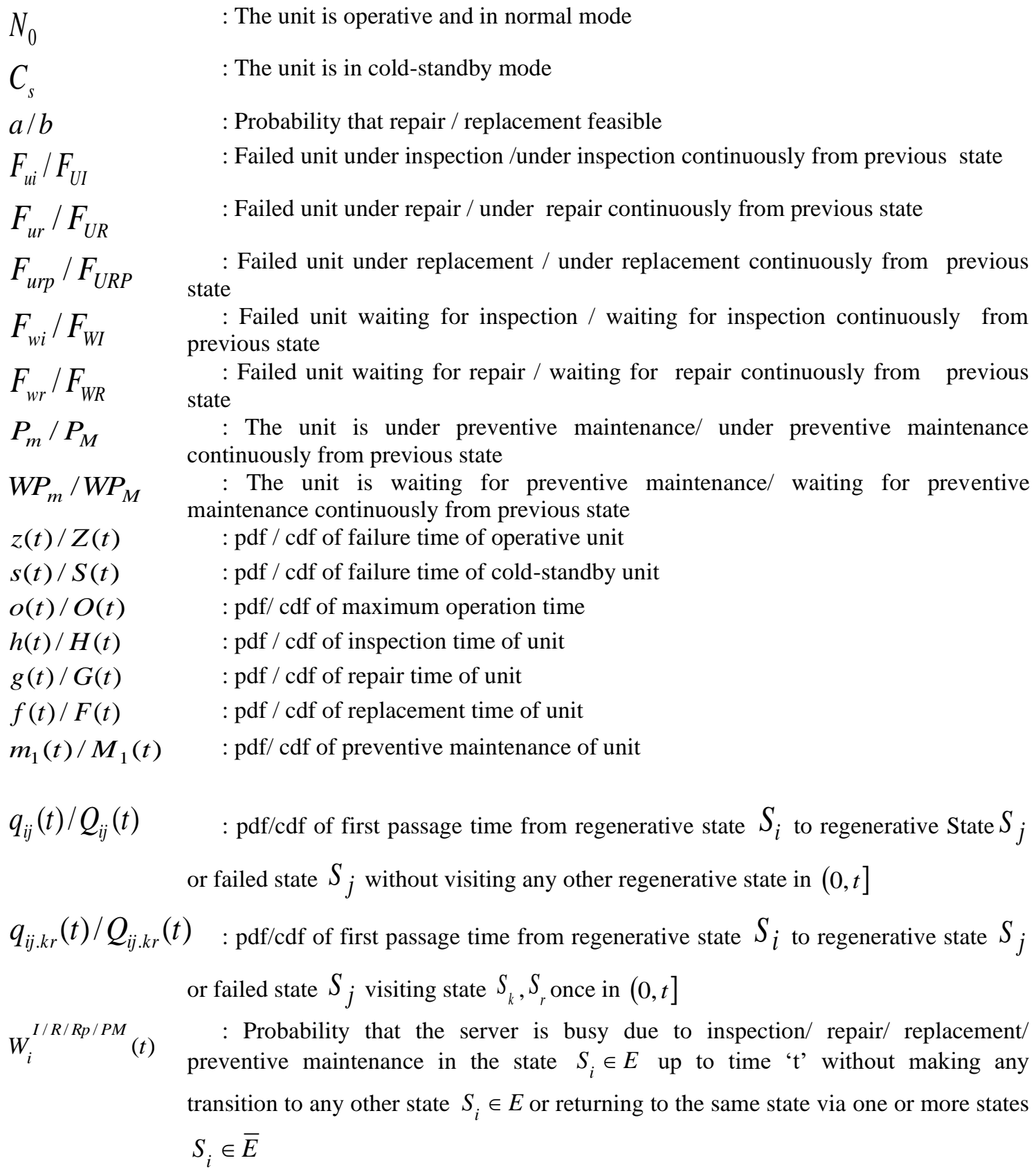

$\mu_{i}(t) \quad:$ Probability that the system up initially in state $S_{i} \in E_{\text {is }}$ up at time t without visiting to any regenerative state

$M_{i}(t) \quad$ Probability that the system up initially in state $S_{i} \in E_{\text {is }}$ up at time t without visiting to any regenerative state

$W_{i}(t) \quad$ : Probability that server busy in the state $S_{i}$ up to time t without making any transition to any other regenerative state or returning to the same state via one or more non-regenerative states

$[s] /[c] \quad:$ Symbol for Laplace-Stietjes convolution/Laplace convolution

$\sim / * \quad:$ Symbol for Laplace- stietjes Transform (LST)/Laplace transform (LT)

$\mathrm{CDF} \quad:$ :Cumulative distribution Function

Pdf : Probability density function

\section{System Description And Assumptions}

1. The system consists of two identical units. There are two modes of the units- operable (Normal) or nonoperable (failed). 
2. At time $\mathrm{t}=0$, the system starts with one unit in active operation mode and another in cold standby.

3. The standby unit instantly switches into operation at the failure of operating unit, putting the failed directly under repair.

4. At the instant the unit in cold standby mode crosses a pre-specified maximum redundancy time failsjust taken for inspectionfor deciding about repair or replacement.

5. The unit in operation taken under preventive maintenance just after crossing a pre-specified time limit, called maximum operation time.

6. The random variables associated with the model follow arbitrary probability distributions.

7. All the repairs and switching are perfect.

\section{Mathematical Description Of The Model}

In this study, a stochastically failing and renewable system is considered. Figure 1 shows the conceptual layout of the system design. Let $S_{i} ; i=0,1,2, \ldots \ldots \ldots \ldots 16$, denote the $i^{\text {th }}$ state of the system. At the time, $t=0$ the system starts operation in the state $S_{0}$. All the possible states are classified into two mutually exclusive and exhaustive categories; regenerative and non-regenerative states. It is observed that $S_{0}, S_{1}, S_{2}, S_{3}$ and $S_{4}$ are regenerative states. If $\tau_{0}, \tau_{1}, \tau_{2}, \ldots \ldots$.. be the time points at which system enters into $S_{i} \in E$, set of regenerative states and $X_{n}$ be the state visited at instant $\tau_{n}+$ i.e. just after the transition at $\tau_{n}$, then $\left\{X_{n}, \tau_{n} ; n \in E\right.$, set of regenerative states $\}$ represent Markov renewal stochastic process. The transition probability matrix of the associated embedded Markov chain is given by $P=\left[P_{i j}\right]=Q_{i j}(\infty)=Q(\infty)$. Here $Q_{i j}(t)$ is the semi-Markov kernel over $E$ such that $Q_{i j}(t)=P\left[X_{n+1}=j, \tau_{n+1}-\tau_{n} \leq t \mid X_{n}=i\right]$, with non-zero elements $P_{i j}$, as follows:

$$
p_{01}=\int_{0}^{\infty} z(t) \bar{O}(t) \bar{S}(t) d t, p_{02}=\int_{0}^{\infty} s(t) \bar{Z}(t) \bar{O}(t) d t, p_{03}=\int_{0}^{\infty} o(t) \bar{S}(t) \bar{Z}(t) d t,
$$$$
p_{10}=\int_{0}^{\infty} g(t) \bar{O}(t) \bar{Z}(t) d t, p_{15}=\int_{0}^{\infty} z(t) \bar{G}(t) \bar{O}(t) d t, p_{16}=\int_{0}^{\infty} o(t) \bar{Z}(t) \bar{G}(t) d t,
$$$$
p_{21}=\int_{0}^{\infty} a h(t) \bar{O}(t) \bar{Z}(t) d t, p_{24}=\int_{0}^{\infty} b h(t) \bar{O}(t) \bar{Z}(t) d t, p_{2,11}=\int_{0}^{\infty} o(t) \bar{H}(t) \bar{Z}(t) d t,
$$$$
p_{2,12}=\int_{0}^{\infty} z(t) \bar{O}(t) \bar{H}(t) d t, p_{30}=\int_{0}^{\infty} m_{1}(t) \bar{O}(t) \bar{Z}(t) d t, p_{37}=\int_{0}^{\infty} z(t) \bar{O}(t) \bar{M}_{1}(t) d t \text {, }
$$$$
p_{38}=\int_{0}^{\infty} o(t) \bar{Z}(t) \bar{M}_{1} d t, p_{40}=\int_{0}^{\infty} f(t) \bar{O}(t) \bar{Z}(t) d t, p_{49}=\int_{0}^{\infty} o(t) \bar{F}(t) \bar{Z}(t) d t,
$$$$
p_{4,10}=\int_{0}^{\infty} z(t) \bar{O}(t) \bar{F}(t) d t, p_{51}=\int_{0}^{\infty} g(t) d t, p_{63}=\int_{0}^{\infty} g(t) d t, p_{71}=\int_{0}^{\infty} m_{1}(t) d t, p_{83}=\int_{0}^{\infty} m_{1}(t) d t,
$$$$
p_{93}=\int_{0}^{\infty} f(t) d t, p_{10,1}=\int_{0}^{\infty} f(t) d t, p_{11,13}=\int_{0}^{\infty} a h(t) d t, p_{11,14}=\int_{0}^{\infty} b h(t) d t, p_{12,15}=\int_{0}^{\infty} b h(t) d t \text {, }
$$$$
p_{12,16}=\int_{0}^{\infty} a h(t) d t, p_{13,3}=\int_{0}^{\infty} g(t) d t, p_{14,3}=\int_{0}^{\infty} f(t) d t, p_{15,1}=\int_{0}^{\infty} f(t) d t, p_{16,1}=\int_{0}^{\infty} g(t) d t \text {, }
$$

$p_{11.5}=p_{15} p_{51,} p_{13.6}=p_{16} p_{63}, p_{21.12,15}=p_{2,12} p_{12,15} p_{15,1}, p_{21.12,16}=p_{2,12} p_{12,16} p_{16,1}$,

$p_{23.11,13}=p_{2,11} p_{11,13} p_{13,3}, p_{23.11,14}=p_{2,11} p_{11,14} p_{14,3}, p_{31.7}=p_{37} p_{71}, p_{33.8}=p_{38} p_{83}$,

$p_{43.9}=p_{49} p_{93}, p_{41.10}=p_{4,10} p_{10,1}$ 
Evidently,

$$
\begin{aligned}
& p_{01}+p_{02}+p_{03}=1, p_{10}+p_{15}+p_{16}=1, p_{21}+p_{24}+p_{2,11}+p_{2,12}=1, p_{30}+p_{37}+p_{38}=1, p_{40}+p_{49}+p_{4,10}=1, \\
& p_{51}=p_{63}=p_{71}=p_{83}=p_{93}=p_{10,1}=1 \text { also } p_{11,13}+p_{11,14}=1, p_{12,15}+p_{12,16}=1, p_{13,3}=p_{14,3}=p_{15,1}=p_{16,1}=1
\end{aligned}
$$

The mean sojourn time in the state $S_{i} \in E$ is given by $\mu_{i}=E(t)=\int_{0}^{\infty} P(T>t) d t$, where $\mathrm{T}$ denotes the time to system failure, such that

$$
\begin{gathered}
\mu_{0}=\int_{0}^{\infty} \bar{O}(t) \bar{Z}(t) \bar{S}(t) d t, \mu_{1}=\int_{0}^{\infty} \bar{O}(t) \bar{G}(t) \bar{Z}(t) d t, \mu_{2}=\int_{0}^{\infty} \bar{O}(t) \bar{H}(t) \bar{Z}(t) d t, \\
\mu_{3}=\int_{0}^{\infty} \bar{O}(t) \bar{M}_{1}(t) \bar{Z}(t) d t, \mu_{4}=\int_{0}^{\infty} \bar{O}(t) \bar{Z}(t) \bar{F}(t) d t
\end{gathered}
$$

The unconditional mean time taken by the system to transit to any regenerative state $S_{j}$ when time is counted from an epoch of entrance into that state $S_{i}$ is given by;

$$
\begin{gathered}
m_{i j}=\int_{0}^{\infty} t d\left\{Q_{i j}(t)\right\} \\
m_{10}+m_{11.5}+m_{13.6}=\mu_{1}^{\prime}, \quad m_{21}+m_{24}+m_{21.12,15}+m_{21.12,16}+m_{23.11,13}+m_{23.11,14}=\mu_{2}^{\prime}, \\
m_{30}++m_{31.7}+m_{33.8}=\mu_{3}^{\prime}, m_{40}+m_{43.9}+m_{41.10}=\mu_{4}^{\prime}
\end{gathered}
$$

All the possible transition states of the system model are as follows:

The regenerative states:

$$
\begin{aligned}
& S_{0}=\left(N_{o}, C_{s}\right), S_{1}=\left(F_{u r}, N_{o}\right), \\
& S_{2}=\left(N_{o}, F_{u i}\right), \quad S_{3}=\left(P_{m}, N_{o}\right), \\
& S_{4}=\left(N_{o}, F_{u r p}\right)
\end{aligned}
$$

The non-regenerative states:

$$
\begin{aligned}
& S_{5}=\left(F_{U R}, F_{w r}\right), S_{6}=\left(F_{U R}, W P_{m}\right), \\
& S_{7}=\left(P_{M}, F_{w r}\right), S_{8}=\left(P_{M}, W P_{m}\right), \\
& S_{9}=\left(W P_{m}, F_{U R p}\right), S_{10}=\left(F_{w r}, F_{U R p}\right), \\
& S_{11}=\left(W P_{m}, F_{U I}\right), S_{12}=\left(F_{w r}, F_{U I}\right), \\
& S_{13}=\left(W P_{M}, F_{u r}\right), S_{14}=\left(W P_{M} F_{u r p}\right), \\
& S_{15}=\left(F_{W R}, F_{u r p}\right), S_{16}=\left(F_{W R}, F_{u r}\right)
\end{aligned}
$$

The expressions for all the measures of system performance can be expressed in terms ofthe transition probabilities and the mean sojourn times.

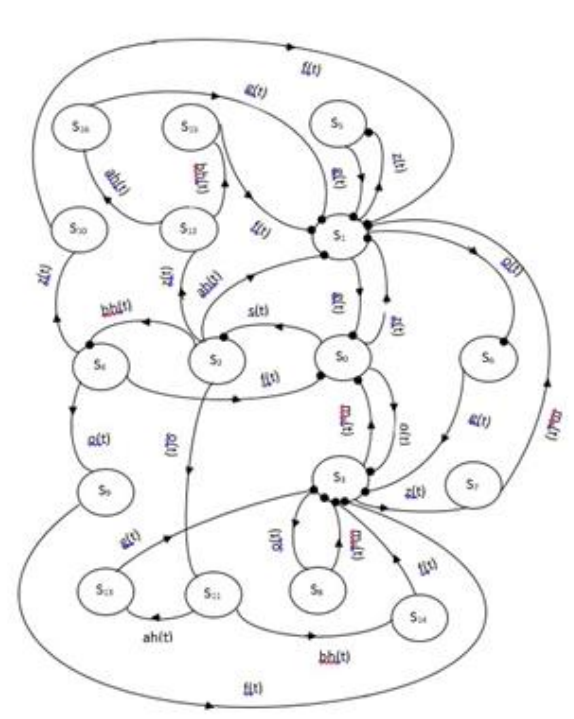




\section{Reliability and Meantime to system failure (MTSF)}

Let $\phi_{i}(t)$ be the cdf of time to system failure i.e. first passage time, starting from the state $S_{i} \in E$ up to a failed state. Using the theory of regenerative processes, we have the following set of recursive relations for $\phi_{i}(t)$

$$
\phi_{i}(t)=\sum_{\substack{i, j \in(E \cap U) \\ k, l, m \ldots \in \bar{U}}}\left\{Q_{i, j}(t)+Q_{i, j . k}(t)+Q_{i, j . k l}(t)+\ldots . .+Q_{i, j . k l m \ldots . . .}(t)\right\}[s] \phi_{j}(t)+\sum_{\substack{i \in(E \cap U) \\ f \in \bar{U}}} Q_{i, f}(t) ; i=0,1,2,3,4
$$

Here, $S_{j}$ is an operative regenerative state to which the given regenerative state $S_{i}$ can transit and $S_{k}$ is a failed state to which the state $S_{i}$ can transit directly. Further, $S_{i, j . k l m \ldots . .}$ denotes the transition of the system from state $S_{i} \in E$ to $S_{j} \in E$ via failed states $S_{k}, S_{l}, S_{m} \ldots \ldots$

For $i=1$, we have

$$
\phi_{1}(t)=Q_{10}(t)[s] \phi_{0}(t)+\sum_{j=5,6} Q_{1 j}(t)
$$

The left-hand side of this equation shows that the system remains operative $S_{1} \in E \cap U$, until time $t$. The first term of right-hand side indicates that the system transits from $S_{1}$ to $S_{0}$ at a time less than time $t$ and the system completes its operation until the time $t$, starting from state $S_{0}$ whereas the second term implies thatthe system moves from a regenerative up-state $S_{1}$ to failed states $S_{5}$ and $S_{6}$. Similarly, all other possible equations can be obtained and explained using above general expression.

Taking LST of above relation (1) and solving for $\widetilde{\phi}_{0}(s)$, omitting the argument s for brevity,we get:

$$
\tilde{\phi}_{0}(s)=\frac{\left[\tilde{Q}_{15}+\tilde{Q}_{16}\right]\left[\tilde{Q}_{01}+\tilde{Q}_{02} \tilde{Q}_{21}\right]+\tilde{Q}_{02}\left[\tilde{Q}_{2,11}+\tilde{Q}_{2,12}\right]+\tilde{Q}_{02} \tilde{Q}_{24}\left[\tilde{Q}_{49}+\tilde{Q}_{4,10}\right]+\tilde{Q}_{03}\left[\tilde{Q}_{37}+\tilde{Q}_{38}\right]}{1-\tilde{Q}_{02} \tilde{Q}_{24} \tilde{Q}_{40}-\tilde{Q}_{03} \tilde{Q}_{30}-\tilde{Q}_{10}\left[\tilde{Q}_{01}+\tilde{Q}_{02} \tilde{Q}_{21}\right]}
$$

The reliability and mean time to system failure (MTSF) are given by

$$
\begin{aligned}
& \text { Reliability } R(t)=L^{-1}\left[\frac{1-\tilde{\phi}_{0}(s)}{s}\right] \\
& M T S F=\lim _{s \rightarrow 0} \frac{1-\tilde{\phi}_{0}(s)}{s}=\frac{N_{1}}{D_{1}}
\end{aligned}
$$

Where

$$
\begin{gathered}
N_{1}=\mu_{0}+\left[p_{01}+p_{02} p_{21}\right] \mu_{1}+p_{02} \mu_{2}+p_{03} \mu_{3}+p_{02} p_{24} \mu_{4} \\
D_{1}=1-p_{01} p_{10}-p_{02}\left[p_{10} p_{21}+p_{24} p_{40}\right]-p_{03} p_{30}
\end{gathered}
$$

\section{Analysis of Economic Measures}

Let the system entered the regenerative state $S_{i} \in E$, at $\mathrm{t}=0$. Considering $S_{j} \in E$, as a regenerative state to which the given regenerative state $S_{i} \in E$, reaches. Now using the above terms the recursive relations for various measures of system performance are given as follows:

\section{a. System availability:}

Let $A_{i}(t)=P\left[S_{i} \in U\right.$, at time $t \mid S_{i} \in E$, at $\left.t=0\right]$, then using the standard notations given in section 2, we have the following expression for the system availability in $(0, \mathrm{t}]$ : 


$$
A_{i}(t)=M_{i \in(U \cap E)}(t)+\sum_{\substack{i, j \in E \\ k, l, m \ldots \in(\bar{U} \cap \bar{E})}}\left[q_{i, j}(t)+\delta_{i, j . k, l \ldots \ldots}\left\{q_{i, j . k}(t)+q_{i, j . k, l}(t)+\ldots \ldots\right\}\right](c) A_{j}(t) ; i=0,1,2,3,4
$$

\section{b. Busy period}

\section{Let us define}

$\mathrm{B}_{\mathrm{i}}^{\mathrm{I} / \mathrm{B} / \mathrm{Rp} / \mathrm{PM}}(\mathrm{t})=\mathrm{P}\left[\right.$ Server is busy in inspection/repair/replacement/PM at time $\mathrm{t} \mid \mathrm{S}_{\mathrm{i}} \in \mathrm{E}$ at $\left.\mathrm{t}=0\right]$

Now using the simple probability rules we have following expressions for different busy periods of the server in $(0, \mathrm{t}]$ :

i. $\quad$ Server busy period due to inspection

$$
B_{i}^{I}(t)=W_{i \in E}^{I}(t)+\sum_{\substack{i, j \in E \\ k, l, m \ldots \in \in(\bar{U} \cap \bar{E})}}\left\{q_{i, j}(t)+\delta_{i, j, k, l \ldots \ldots . . .}\left\{q_{i, j . k}(t)+q_{i, j . k l}(t)+\ldots \ldots . .\right\}\right\}(c) B_{j}^{I}(t) ; i=0,1,2,3,4
$$

ii. $\quad$ Server busy period due to repair

$$
B_{i}^{R}(t)=W_{i \in E}^{R}(t)+\sum_{\substack{i, j \in E \\ k, l, m \ldots . \in(\bar{U} \cap \bar{E})}}\left\{q_{i, j}(t)+\delta_{i, j, k, l \ldots \ldots . . .}\left\{q_{i, j . k}(t)+q_{i, j . k l}(t)+\ldots \ldots . .\right\}\right\}(c) B_{j}^{R}(t) ; i=0,1,2,3,4
$$

iii. $\quad$ Server busy period due to replacement

$$
B_{i}^{R p}(t)=W_{i \in E}^{R p}(t)+\sum_{\substack{i, j \in E \\ k, l, m \ldots \in \in(\bar{U} \cap \bar{E})}}\left\{q_{i, j}(t)+\delta_{i, j, k, l \ldots \ldots . .}\left\{q_{i, j . k}(t)+q_{i, j . k l}(t)+\ldots \ldots . .\right\}\right\}(c) B_{j}^{R p}(t) ; i=0,1,2,3,4
$$

iv. Server busy period due to preventive maintenance

$$
B_{i}^{P M}(t)=W_{i \in E}^{P M}(t)+\sum_{\substack{i, j \in E \\ k, l, m \ldots \ldots \in(\bar{U} \cap \bar{E})}}\left\{q_{i, j}(t)+\delta_{i, j, k, l \ldots \ldots . . .}\left\{q_{i, j . k}(t)+q_{i, j . k l}(t)+\ldots \ldots . .\right\}\right\}(c) B_{j}^{P M}(t) ; \quad i=0,1,2,3,4
$$

\section{c. Expected number of remedial activities}

Let us first define the conditional expectation of the remedial activities done on the system i.e.

$I_{i}(t) / R_{i}(t) / R_{i}^{C}(t) / P_{i}^{M}(t)=E\left[N o\right.$ of insp./repairs/repl./PM of unit in $(0, \mathrm{t}] \mid \mathrm{S}_{\mathrm{i}} \in \mathrm{E}$ at time $\left.\mathrm{t}=0\right]$ Now using some probabilistic rules we have the following expressions:

$i$. Expected number of inspections of the unit

$$
I_{i}(t)=\sum_{\substack{i, j \in E \\ k, l, m \ldots . \in(\bar{U} \cap \bar{E})}}\left\{Q_{i, j}(t)+\delta_{i, j . k l \ldots \ldots . .}\left\{Q_{i, j . k}(t)+Q_{i, j . k l}(t)+\ldots \ldots . .\right\}\right\}(s)\left\{\delta_{j}+I_{j}(t)\right\} ; \quad i=0,1,2,3,4
$$

ii. $\quad$ Expected number of repairs of the unit

$$
R_{i}(t)=\sum_{\substack{i, j \in E \\ k, l, m \ldots \ldots \in(\bar{U} \cap \bar{E})}}\left\{Q_{i, j}(t)+\delta_{i, j . k l \ldots \ldots . . .}\left\{Q_{i, j . k}(t)+Q_{i, j . k l}(t)+\ldots \ldots . .\right\}\right\}(s)\left\{\delta_{j}+R_{j}(t)\right\} ; \quad i=0,1,2,3,4
$$

iii. $\quad$ Expected number of replacements of the unit

$$
R_{i}^{C}(t)=\sum_{\substack{i, j \in E \\ k, l, m \ldots \in(\bar{U} \cap \bar{E})}}\left\{Q_{i, j}(t)+\delta_{i, j . k l \ldots \ldots . . .}\left\{Q_{i, j . k}(t)+Q_{i, j . k l}(t)+\ldots \ldots . .\right\}\right\}(s)\left\{\delta_{j}+R_{i}^{C}(t)\right\} ; \quad i=0,1,2,3,4
$$

iv. Expected number of preventive maintenance of the unit

$$
P_{i}^{M}(t)=\sum_{\substack{i, j \in E \\ k, l, m \ldots \in \in(\bar{U} \cap \bar{E})}}\left\{Q_{i, j}(t)+\delta_{i, j . k l \ldots \ldots . .}\left\{Q_{i, j . k}(t)+Q_{i, j . k l}(t)+\ldots \ldots . .\right\}\right\}(s)\left\{\delta_{j}+P_{i}^{M}(t)\right\} ; \quad i=0,1,2,3,4
$$


Here $\delta_{j}= \begin{cases}1 \text {; if there is a repair/ treatment from } \mathrm{S}_{\mathrm{i}} \text { to } \mathrm{S}_{\mathrm{j}} \\ 0 ; & \text { Otherwise }\end{cases}$

And $\delta_{i, j . k . l \ldots \ldots . . .}=\left\{\begin{array}{cc}1 \text {; if there is a transition from } \mathrm{S}_{\mathrm{i}} \text { to } \mathrm{S}_{\mathrm{j}} \text { via } \mathrm{S}_{\mathrm{k}, 1, \ldots \ldots . .} \\ 0 ; & \text { Otherwise }\end{array}\right.$

\section{Steady-State Analysis}

To obtain the steady results we use the method of Laplace/ Laplace-Stieltjes transform. The Laplace-Stieltjes transform of a real-valued function $\mathrm{f}$ is given by

$$
L[f(t)]=\int e^{-s t} d f(t)=f(s)
$$

The final value formula of Laplace transform states that

$\lim _{t \rightarrow \infty} f(t)=\lim _{s \rightarrow 0} s f(s)$

Using these formulae, we obtained the expressions for following system performance indices:

$$
\begin{aligned}
& A_{0}=\lim _{t \rightarrow \infty} A_{0}(t)=\lim _{s \rightarrow 0} s L\left[A_{0}(t)\right]=\frac{N_{2}}{D_{2}} ; \quad B_{0}^{I}=\lim _{t \rightarrow \infty} B_{0}^{I}(t)=\lim _{s \rightarrow 0} s L\left[B_{0}^{I}(t)\right]=\frac{N_{3}^{I}}{D_{2}} ; \\
& B_{0}^{R}=\lim _{t \rightarrow \infty} B_{0}^{R}(t)=\lim _{s \rightarrow 0} s L\left[B_{0}^{R}(t)\right]=\frac{N_{3}^{R}}{D_{2}} ; \quad B_{0}^{R p}=\lim _{t \rightarrow \infty} B_{0}^{R p}(t)=\lim _{s \rightarrow 0} s L\left[B_{0}^{R p}(t)\right]=\frac{N_{3}^{R p}}{D_{2}} ; \\
& B_{0}^{P M}=\lim _{t \rightarrow \infty} B_{0}^{P M}(t)=\lim _{s \rightarrow 0} s L\left[B_{0}^{P M}(t)\right]=\frac{N_{3}^{P M}}{D_{2}} ; \quad I_{0}=\lim _{t \rightarrow \infty} I_{0}(t)=\lim _{s \rightarrow 0} s L\left[I_{0}(t)\right]=\frac{N_{4}^{I}}{D_{2}} \\
& R_{0}=\lim _{t \rightarrow \infty} R_{0}(t)=\lim _{s \rightarrow 0} s L\left[R_{0}(t)\right]=\frac{N_{4}^{R}}{D_{2}} ; \quad R_{0}^{C}=\lim _{t \rightarrow \infty} R_{0}^{C}(t)=\lim _{s \rightarrow 0} s L\left[R_{0}^{C}(t)\right]=\frac{N_{4}^{C}}{D_{2}} ; \\
& P_{0}^{M}=\lim _{t \rightarrow \infty} P_{0}^{M}(t)=\lim _{s \rightarrow 0} s L\left[P_{0}^{M}(t)\right]=\frac{N_{4}^{P M}}{D_{2}} \\
& N_{2}=\left[\mu_{0}+\mu_{2} p_{02}+\mu_{4} p_{24} p_{02}\right]\left\{p_{10}\left[1-p_{33.8}\right]+p_{13.6} p_{30}\right\}+\mu_{1}\left\{p _ { 0 2 } \left[p_{24}\left(p_{30} p_{41.10}-p_{40} p_{31.7}\right)\right.\right. \\
& \left.\left.+p_{30}\left(p_{21}+p_{21.12,15}+p_{21.12,16}\right)\right]+p_{01} p_{30}+p_{31.7}\right\}+\mu_{3}\left[p _ { 0 2 } \left\{p_{10}\left(p_{23.11,13}+p_{23.11,14}+p_{24} p_{43.9}\right)\right.\right. \\
& \left.\left.-p_{24} p_{40} p_{13.6}\right\}+p_{13.6}+p_{03} p_{10}\right] \\
& D_{2}=\left[\mu_{0}+\mu_{2}^{\prime} p_{02}+\mu_{4}^{\prime} p_{24} p_{02}\right]\left\{p_{10}\left[1-p_{33.8}\right]+p_{13.6} p_{30}\right\}+\mu_{1}^{\prime}\left\{p _ { 0 2 } \left[p_{24}\left(p_{30} p_{41.10}-p_{40} p_{31.7}\right)\right.\right. \\
& \left.\left.+p_{30}\left(p_{21}+p_{21.12,15}+p_{21.12,16}\right)\right]+p_{01} p_{30}+p_{31.7}\right\}+\mu_{3}^{\prime}\left[p _ { 0 2 } \left\{p_{10}\left(p_{23.11,13}+p_{23.11,14}+p_{24} p_{43.9}\right)\right.\right. \\
& \left.\left.-p_{24} p_{40} p_{13.6}\right\}+p_{13.6}+p_{03} p_{10}\right] \\
& N_{3}^{R}=W_{1}^{R^{*}}(0)\left[1-p_{33.8}\right]\left[p_{02}\left\{p_{24} p_{41.10}+p_{21}+p_{21.12,15}+p_{21.12,16}\right\}+p_{01}\right]+W_{1}^{R^{*}}(0) p_{31.7}\left[p _ { 0 2 } \left\{p_{24} p_{43.9}\right.\right. \\
& \left.\left.+p_{23.11,13}+p_{23.11,14}\right\}+p_{03}\right] \\
& N_{3}^{R p}=W_{4}^{R p^{*}}(0) p_{02} p_{24}\left\{p_{10}\left[1-p_{33.8}\right]+p_{13.6} p_{30}\right\}, N_{3}^{I}=W_{2}^{I^{*}}(0) p_{02}\left\{p_{10}\left[1-p_{33.8}\right]+p_{13.6} p_{30}\right\} \\
& N_{3}^{P M}=W_{3}^{P M^{*}}(0)\left[1-p_{11.5}\right]\left\{p_{02}\left[p_{23.11,13}+p_{23.11,14}+p_{24} p_{43.9}\right]+p_{03}\right\}+W_{3}^{P M^{*}}(0) p_{13.6}\left\{p _ { 0 2 } \left[p_{21}+p_{21.12,15}\right.\right. \\
& \left.\left.+p_{21.12,16}+p_{24} p_{41.10}\right]+p_{01}\right\} \\
& N_{4}^{R}=\left[1-p_{33.8}\right]\left\{p_{02} p_{24} p_{41.10}+p_{02}\left[1-p_{24}\right]+p_{01}+p_{02} p_{10}\left[p_{21.12,16}+p_{23.11,13}\right]\right\}+p_{02} p_{24} p_{31.7} p_{43.9} \\
& -p_{02} p_{30}\left[p_{23.11,13}+p_{23.11,14}\right]+p_{03} p_{31.7}+\left[p_{21.12,16}+p_{23.11,13}\right] p_{02} p_{13.6} p_{30} \\
& N_{4}^{C}=p_{02}\left\{p_{10}\left[1-p_{33.8}\right]+p_{13.6} p_{30}\right\}\left[p_{24}+p_{21.12,15}+p_{23.11,14}\right], N_{4}^{I}=p_{02}\left\{p_{10}\left[1-p_{33.8}\right]+p_{13.6} p_{30}\right\}
\end{aligned}
$$




$$
\begin{aligned}
N_{4}^{P M}= & {\left[1-p_{11.5}\right]\left\{p_{02}\left[p_{23.11,13}+p_{23.11,14}+p_{24} p_{43.9}\right]+p_{03}\right\}+p_{13.6}\left\{p _ { 0 2 } \left[p_{21}+p_{21.12,15}+p_{21.12,16}\right.\right.} \\
& \left.\left.+p_{24} p_{41.10}\right]+p_{01}\right\}
\end{aligned}
$$

Now we can obtain the profit incurred to the system in steady-state viz.

Profit $=($ total revenue generated $)-($ total expenses occured $)$

$P_{0}=\left(K_{0} A_{0}\right)-\left(K_{1} B_{0}^{I}+K_{2} B_{0}^{R}+K_{3} B_{0}^{R p}+K_{4} I_{0}+K_{5} R_{0}+K_{6} R_{0}^{C}+K_{7} B_{0}^{P M}+K_{8} P_{0}^{M}\right)$

$K_{0}=$ Revenue per unit up-time of the system

$K_{1}=$ Cost per unit time for which server is busy in the inspection of the standby

$K_{2}=$ Cost per unit time for which server is busy due to repair

$K_{3}=$ Cost per unit time for which server is busy due to replacement

$K_{4}=$ Cost per unit inspection

$K_{5}=$ Cost per unit repair

$K_{6}=$ Cost per unit replacement

$K_{7}=$ Cost per unit time for which server is busy due to preventive maintenance

$K_{8}=$ Cost per unit preventive maintenance

\section{Graphical Illustrations}

\section{a. Case-1: Weibull Distribution}

Taking shape parameter $\eta=0.5$, the pdf for different random variables becomes as follows:

$$
\begin{aligned}
& z(t)=\frac{\lambda}{2 \sqrt{t}} \exp (-\lambda \sqrt{t}), s(t)=\frac{\mu}{2 \sqrt{t}} \exp (-\mu \sqrt{t}), o(t)=\frac{\xi}{2 \sqrt{t}} \exp (-\xi \sqrt{t}), \\
& g(t)=\frac{\beta}{2 \sqrt{t}} \exp (-\beta \sqrt{t}), \\
& f(t)=\frac{\gamma}{2 \sqrt{t}} \exp (-\gamma \sqrt{t}), h(t)=\frac{\alpha}{2 \sqrt{t}} \exp (-\alpha \sqrt{t}), m_{1}(t)=\frac{v}{2 \sqrt{t}} \exp (-v \sqrt{t}),
\end{aligned}
$$

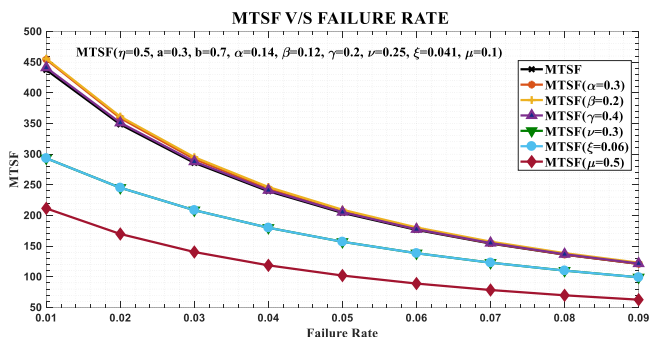

Fig. 1: Effect of various parameters on MTSF $(\eta=0.5)$

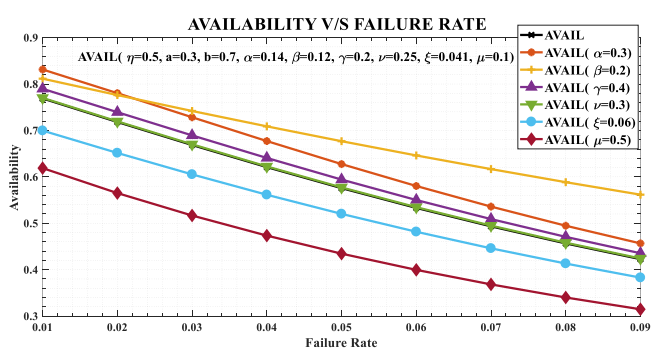

Fig. 2: Effect of various parameters on Availability $(\eta=0.5)$ 


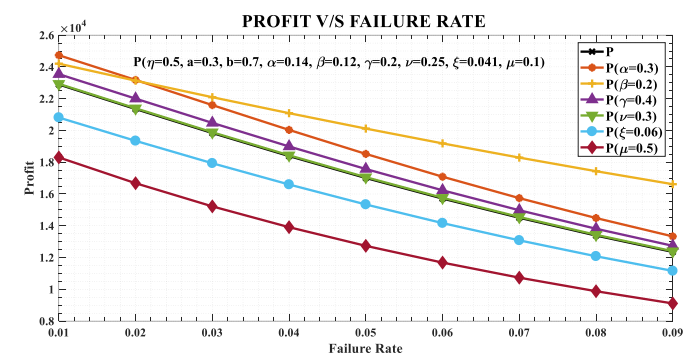

Fig. 3: Effect of various parameters on Profit $(\eta=0.5)$

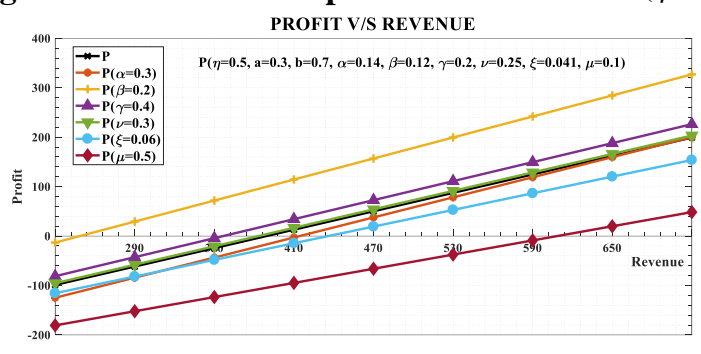

Fig. 4: Effect of various parameters on Profit $(\eta=0.5)$

\section{b. Case-2: Exponential Distribution}

Taking shape parameter $\eta=1.0$, the pdf for different random variables becomes as follows $z(t)=\lambda \exp (-\lambda t), s(t)=\mu \exp (-\mu t), o(t)=\xi \exp (-\xi t), g(t)=\beta \exp (-\beta t)$, $f(t)=\gamma \exp (-\gamma t), h(t)=\alpha \exp (-\alpha t), m_{1}(t)=v \exp (-v t)$.

where $\mathrm{t} \geq 0$ and $\eta, \lambda, \mu, v, \beta, \gamma, \alpha, \xi>0$

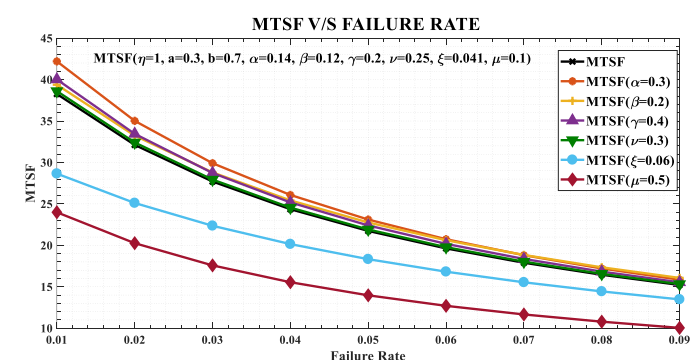

Fig. 5: Effect of various parameters on MTSF $(\eta=1)$

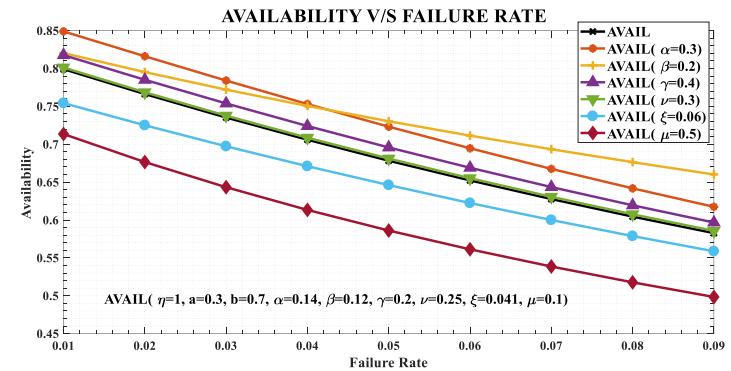

Fig. 6: Effect of various parameters on Availability $(\eta=1)$

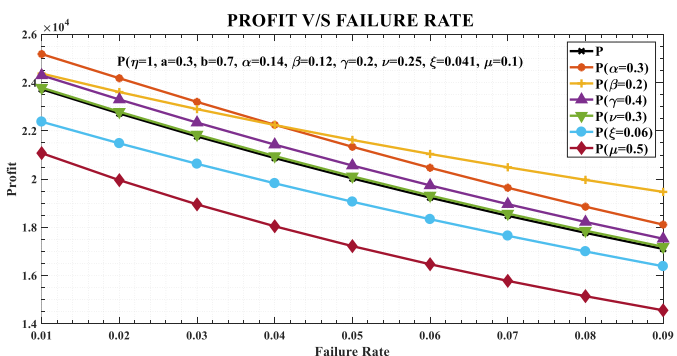


Fig. 7: Effect of various parameters on Profit $(\eta=1)$

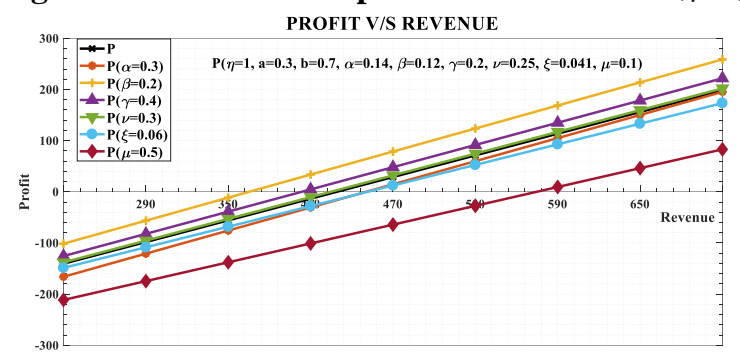

Fig. 8: Effect of various parameters on Profit $(\eta=1)$

\section{c. Case-3: Rayleigh Distribution}

When shape parameter $\eta=2$ then the probability distributions reduce to Rayleigh with the pdfs given below:

$$
z(t)=2 \lambda t \exp \left(-\lambda t^{2}\right), s(t)=2 \mu t \exp \left(-\mu t^{2}\right), o(t)=2 \xi t \exp \left(-\xi t^{2}\right), g(t)=2 \beta t \exp \left(-\beta t^{2}\right),
$$

$f(t)=2 \gamma t \exp \left(-\lambda^{2}\right), h(t)=2 \alpha t \exp \left(-\alpha t^{2}\right), m_{1}(t)=2 v t \exp \left(-v t^{2}\right)$,

where $\mathrm{t} \geq 0$ and $\eta, \lambda, \mu, \xi, \beta, \gamma, \alpha, v>0$

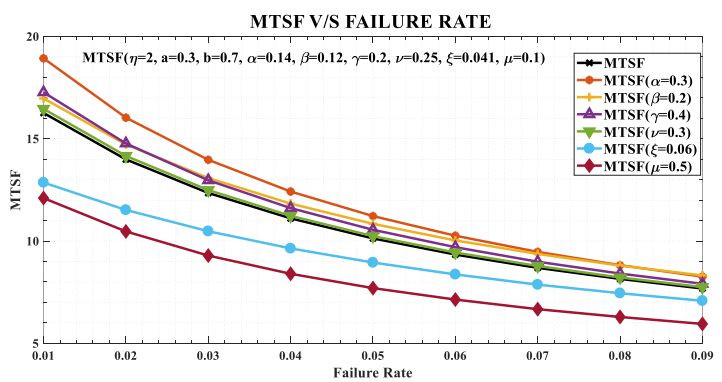

Fig. 9: Effect of various parameters on MTSF $(\eta=2)$

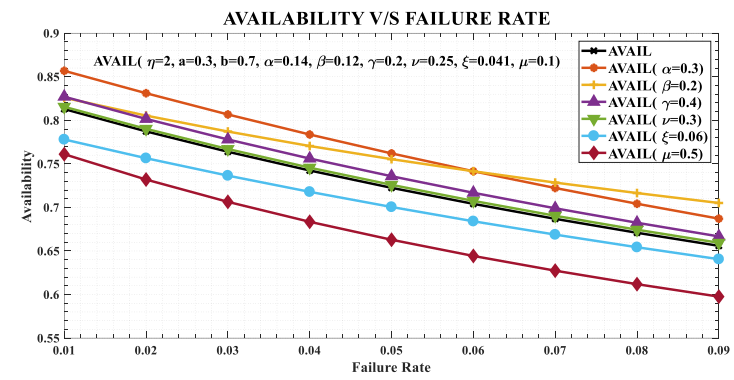

Fig. 10: Effect of various parameters on Availability $(\eta=2)$

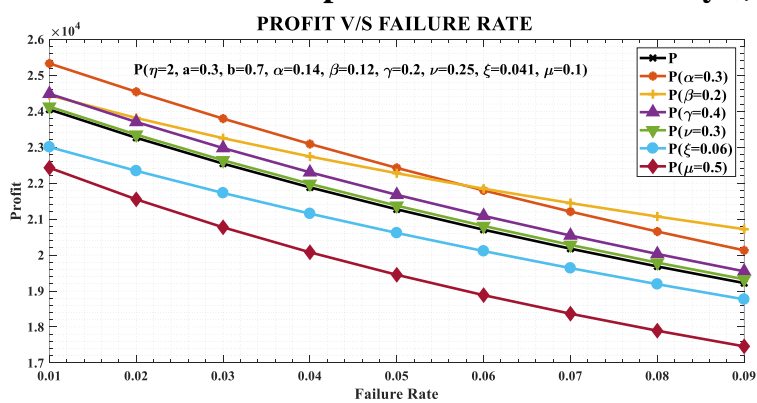

Fig. 11: Effect of various parameters on Profit $(\eta=2)$ 


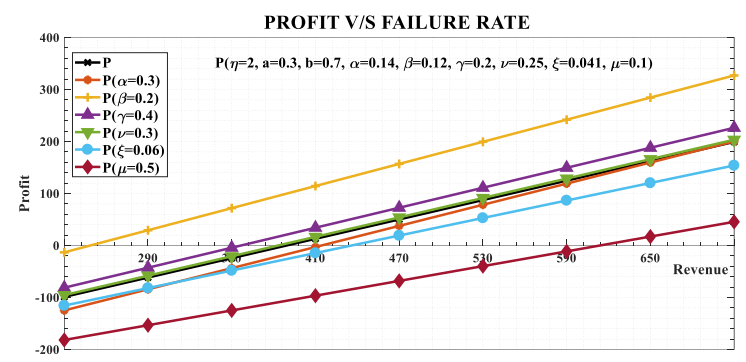

Fig. 12: Effect of various parameters on Profit $(\eta=2)$

\section{Discussion On Results}

To carry out above graphical study all the random variables are supposed to follow Weibull distribution and the following data set is considered:

$\mathrm{a}=0.3, \quad \mathrm{~b}=0.7, \quad \alpha=0.14, \quad \beta=0.12, \quad \gamma=0.2, \quad \delta=0.07, \quad \mu=0.1, \quad v=0.25, \quad \xi=0.041, \quad K_{0}=30000, \mathrm{~K}_{1}=100$, $\mathrm{K}_{2}=500, \mathrm{~K}_{3}=150, \mathrm{~K}_{4}=800, \mathrm{~K}_{5}=1000, \mathrm{~K}_{6}=700, \mathrm{~K}_{7}=300, \mathrm{~K}_{8}=400$.

The system performance is studied for three different values of the shape parameter of Weibull distribution that exhibit three distinct probability distributions as follows:

1) $0<\eta<1 ; \eta=0.5$, decreasing failure rate (DFR), fig. $1-4$.

2) $\eta=1$, constant failure rate (CFR)i.e.case of exponential distribution, fig.5-8.

3) $\eta>1 ; \eta=2$, increasing failure rate i.e. case of Ryleigh distribution, fig.9-12.

In all three cases, we observed that MTSF, Availability and Profit declines with increasing value of failure rate. When we fixed the values of other parameters, the values of all these indices go up as value of any of the parameters viz. inspection rate; $\alpha=0.14$ to $\alpha=0.3$, repair rate; $\beta=0.12$ to $\beta=0.2$, replacement rate; $\gamma=0.2$ to $\gamma=0.4$ or the preventive maintenance rate; $v=0.25$ to $v=0.3$ increases. All these indices declines with increasing value of $\mu$ (Maximum redundancy time) and rate at which operative unit goes under preventive maintenance (maximum operation time). Figure 4, 8 and 12 gives the cutoff points for profit against revenue per unit up time of system. In case of $\eta=0.5$, the trend lines of $\alpha=0.3$ decline sharply than the lines corresponding to $\beta=0.2$ for $\lambda>0.02$. It means that replacement works better as compare to inspection after failure rate 0.02 . In case of $\eta=1$, the trend lines of $\alpha=0.3$ decline sharply than the lines corresponding to $\beta=0.2$ for $\lambda>0.04$. In case of $\eta=2$, the trend lines of $\alpha=0.3$ decline sharply than the lines corresponding to $\beta=0.2$ for $\lambda>0.06$.

\section{Conclusion and Future Directions}

This paper analyzed a two-unit cold standby system with limits on maximum redundancy time for the standby and maximum operation time for the operative unit. The discrete state-continuous time Markov regenerative processes are used to develop and analyze the model. The standby unit went under inspection for checking the practicability of repair or replacement after surpassing the maximum redundancy time limit. On the other hand, preventive maintenance is used on the operative unit just after crossing the maximum operation time limit. The grapgical trends obtained for various measures of system performance underlined the proven facts thatsystem performance declines with failures and uprises with remedial activities, that advocate for the model'spracticality.

For future research, the prospective option may be the generalization of this model considering m-operative and $n$-standby components. The assumption of independence of all random variables maybe relexed for more realism.

\section{References}

1. R. D. Yearout, P. Reddy, and D. L. Grosh, "Standby Redundancy in Reliability - A Review," IEEE Trans. Reliab., vol. 35, no. 3, pp. 285-292, 1986, doi: 10.1109/TR.1986.4335434.

2. G. S. Mokaddis, M. L. Tawfek, and S. A. M. Elhssia, "Analysis of a two-dissimilar unit cold standby redundant system subject to inspection and random change in units," Microelectron. Reliab., vol. 37, no. 2, pp. 329-334, Feb. 1997, doi: 10.1016/0026-2714(95)00207-3.

3. M. Manglik and M. Ram, "Reliability analysis of a two unit cold standby system using markov 
process," academia.edu, vol. 6, no. 2, pp. 65-80, 2013, Accessed: Aug. 18, 2020. [Online]. Available: https://www.academia.edu/download/45539094/BASU_05_jrss62.pdf.

4. Y. Chen, Z. Wang, Y. Y. Li, R. Kang, and A. Mosleh, "Reliability analysis of a cold-standby system considering the development stages and accumulations of failure mechanisms," Reliab. Eng. Syst. Saf., vol. 180, pp. 1-12, 2018, doi: 10.1016/j.ress.2018.06.022.

5. H. Jia, G. Levitin, Y. Ding, and Y. Song, "Reliability analysis of standby systems with multi-state elements subject to constant transition rates," Qual. Reliab. Eng. Int., vol. 35, no. 1, pp. 318-328, Feb. 2019, doi: 10.1002/qre.2401.

6. A. K. Dhankar, B. R.K., and S. C. Malik, "Reliability modeling and profit analysis of a system with different failure modes and replaceable server subject to inspection," Int. J. Stat. Anal., vol. 2, no. 3, pp. 245-255, 2012, Accessed: Jan. 31, 2021. [Online]. Available: http://www.ripublication.com/ijsa.htm.

7. R. K. Bhardwaj and R. Singh, "A Cold-Standby System with Server Failure and Delayed Treatment," Int. J. Comput. Appl., vol. 124, no. 17, pp. 31-36, 2015, doi: 10.5120/ijca2015905823.

8. S. Osaki and T. Nakagawa, "On a Two-Unit Standby Redundant System with Standby Failure," Oper. Res., vol. 19, no. 2, pp. 510-523, Apr. 1971, doi: 10.1287/opre.19.2.510.

9. C. Shekhar, A. Kumar, and S. Varshney, "Load sharing redundant repairable systems with switching and reboot delay," Reliab. Eng. Syst. Saf., vol. 193, p. 106656, Jan. 2020, doi: 10.1016/j.ress.2019.106656.

10. R. K. Bhardwaj, K. Kaur, and S. C. Malik, "Reliability indices of a redundant system with standby failure and arbitrary distribution for repair and replacement times," Int. J. Syst. Assur. Eng. Manag., vol. 8, no. 2, pp. 423-431, Jun. 2017, doi: 10.1007/s13198-016-0445-z.

11. M. A. W. Mahmoud and M. E. Moshref, "On a two-unit cold standby system considering hardware, human error failures and preventive maintenance," Math. Comput. Model., vol. 51, no. 5-6, pp. 736745, 2010, doi: 10.1016/j.mcm.2009.10.019.

12. S. C. Malik and S. K. Barak, "Reliability measures of a cold standby system with preventive maintenance and repair," Int. J. Reliab. Qual. Saf. Eng., vol. 20, no. 6, Dec. 2013, doi: 10.1142/S0218539313500228.

13. J. Hu, J. Shen, and L. Shen, "Periodic preventive maintenance planning for systems working under a Markovian operating condition," Comput. Ind. Eng., vol. 142, p. 106291, Apr. 2020, doi: 10.1016/j.cie.2020.106291.

14. W. L. Smith, "Regenerative stochastic processes," in Mathematical and Physical Sciences, Oct. 1955, vol. 232, no. 1188 , pp. 6-31, doi: 10.1098/rspa.1955.0198.

15. K. Sigman and R. W. Wolff, "Review of regenerative processes," SIAM Rev., vol. 35, no. 2, pp. 269288, Jul. 1993, doi: 10.1137/1035046.

16. R. W. Wolff, "Regenerative Processes," in Wiley StatsRef: Statistics Reference Online, John Wiley \& Sons, Ltd, 2014.

17. R. J. D. N. Prabhakar Murthy, Min Xie, Weibull Models, 1st ed. Hoboken, N.J. Wiley-Interscience, 2004.

18. R. B. Abernethy, The New Weibull Handbook, Fifth Ed. R.B. Abernethy, 2010. 